Inorg. Chem. 2020, 59, 18402-18406

doi 10.1021/acs.inorgchem.0c03043

\title{
On the spin-state dependence of redox potentials of spin crossover complexes
}

Dedicated to Dr. Jean-René Hamon on the occasion of his $65^{\text {th }}$ birthday.

Isabelle M. Dixon, ${ }^{\dagger} *$ Sylvain Rat, ${ }^{\ddagger}$ Alix Sournia-Saquet, ${ }^{\ddagger}$ Gábor Molnár, ${ }^{\ddagger}$ Lionel Salmon, ${ }^{\ddagger}$ Azzedine Bousseksou ${ }^{\ddagger} *$

${ }^{\dagger}$ Laboratoire de Chimie et Physique Quantiques, UMR 5626 CNRS/Université Toulouse 3-Paul Sabatier, Université de Toulouse, 118 route de Narbonne, 31062 Toulouse, France

Laboratoire de Chimie de Coordination, UPR 8241, CNRS and Université de Toulouse, UPS, INP, 205 route de Narbonne, 31077 Toulouse, France

* isabelle.dixon@irsamc.ups-tlse.fr, azzedine.bousseksou@1cc-toulouse.fr

\begin{abstract}
Resistance switching properties of nanoscale junctions of spin crossover molecules have received recently much interest. In many cases, this property has been traced back to the variation of molecular orbital energies upon the spin transition. However, one can also expect a substantial reorganization of the molecular structure due to charge localization, which calls for a
\end{abstract}


better understanding of the relationship between the redox potential and the spin state of the molecule. To investigate this issue we carried out a detailed density functional theory and variable temperature cyclic voltammetry investigation of the benchmark compound $\left[\mathrm{Fe}\left(\mathrm{HB}(1,2,4-\text { triazol-1-yl })_{3}\right)_{2}\right]$ in solution. We show that for a correct thermodynamical picture, it is necessary to take into account the charge transfer-induced electronic and structural reorganization as well as spin-equilibria in the oxidized and reduced species.

KEYWORDS Spin crossover, Redox potential, Density functional theory, Electronic Structure, Cyclic voltammetry. 
Spin crossover ( $\mathrm{SCO}$ ) compounds of $3 d^{4}-3 d^{7}$ transition metal complexes display a reversible switching between the low spin (LS) and high spin (HS) electronic states of the central metal ion, which is associated with a remarkable change of the molecular geometry and various physical properties (electrical, optical, magnetic, caloric, mechanical, etc.). ${ }^{1,2}$ Consequently, the SCO phenomenon provides an appealing scope for the modulation of charge and spin transport in microelectronic circuits, which has been explored recently in various test devices. ${ }^{3-7}$

At the macroscopic scale, the dominant transport mechanism in SCO materials is charge carrier hopping via localized electronic states near the Fermi level, which corresponds, in essence, to redox events between neighboring molecules. For this reason, the "bulk" conduction is generally associated with low mobility and pronounced thermal activation. ${ }^{8}$ In the opposite size limit, charge transport is governed by direct tunneling from one electrode to the other. This mechanism is limited by the localization of the electron wavefunction to junctions below ca. 5 nm thickness. ${ }^{9}$ This mode of charge transport refers primarily to single molecule SCO junctions, which have been mostly investigated by scanning tunneling microscopy (STM) and density functional theory (DFT). ${ }^{6,7}$ In most cases, the HS state appears more conducting, which was ascribed to the sizeable decrease of the HOMO-LUMO gap when the ligand field decreases. ${ }^{10,11}$ We shall note, however, that SCO in molecular junctions was achieved in general when strong electronic interactions between the electrode(s) and the molecule(s) were prevented. ${ }^{12,13}$ In this weak coupling limit, a simple orbital-alignment scheme becomes questionable as the junction exhibits Coulomb blockade behavior. ${ }^{14}$ Besides these two size limits (sic bulk and molecular), there have been several reports on SCO devices operating in the "intermediate" range of charge transport (between ca. 5 and $50 \mathrm{~nm}$ ), ${ }^{3}$ wherein multistep tunneling, field emission, directed inelastic hopping, and other mechanisms may occur. Overall, it appears that, apart from the case 
of coherent tunneling, redox events in SCO junctions represent a fundamental feature. Hence, understanding the relationship between the redox potential and the spin state of the molecule is of primary importance towards the analysis of charge transport properties.

In the past, this relationship has been investigated mostly in the context of electron transfer processes accompanied by a change of the spin-state of the metal ion, ${ }^{15}$ which are of particular relevance for a variety of biochemical reactions ${ }^{16-18}$ and electrochemical devices. ${ }^{19-22}$ Obviously, one of the most important factors determining mutually the redox potential, HOMO-LUMO levels and the spin state of transition metal complexes is the ligand field and there is an extensive literature dealing with these relationships among different complexes (for a recent example see ref. 23). On the other hand, due primarily to experimental reasons, relatively few reports have been published on the concomitant investigation of redox and spin-crossover properties of the same complex. ${ }^{24-29}$ Yet, this provides a unique opportunity to investigate the influence of electronic effects on the redox potential, without the additional complexity brought into by ligand substitution. Notably, Kadish et al. ${ }^{26-29}$ reported on a series of cyclic voltammetry (CV) experiments with different ferrous and ferric SCO complexes in solution allowing them to discuss mechanisms relating spin equilibrium and electrochemical reduction and/or oxidation. However, in CV measurements the kinetics of spin-exchange reactions near room temperature is so fast (rate constants range typically between $\left.10^{6}-10^{9} \mathrm{~s}^{-1}\right)^{30}$ that they remain in equilibrium relative to electrochemical events (at typical voltammetric sweep rates). ${ }^{15}$ This means that the standard reduction and oxidation potentials cannot be experimentally determined in both spin states. Yet, voltammetry remains a valuable tool to verify the validity of redox potentials calculated by quantum chemistry methods. 
In this work, we used a DFT approach, which is the most commonly used computational approach in the SCO field, ${ }^{31-36}$ to study the redox reactions of the HS and LS states of [Fe(HB(1,2,4-triazol-1-yl) $\left.)_{3}\right)_{2}$ (compound 1). We have chosen this compound because it exhibits spin-equilibrium in solution near room temperature (allowing thus for $\mathrm{CV}$ experiments) ${ }^{37}$ as well as for its remarkable resistance switching behavior in nanoscale junctions - recently demonstrated in different test devices. ${ }^{38,39}$

\section{Results and Discussion}

As shown in the Kohn-Sham MO diagrams of the neutral species of 1 (Figure 1), the HOMOLUMO gap significantly decreases from ${ }^{1} \mathrm{LS}(4.4 \mathrm{eV})$ to ${ }^{5} \mathrm{HS}(2.8 \mathrm{eV})$, in agreement with previous experimental and theoretical data on various SCO molecules. ${ }^{710-13}$ The intermediate spin species has an intermediate gap ( $3 \mathrm{eV}$, Figure $\mathrm{S} 1)$.
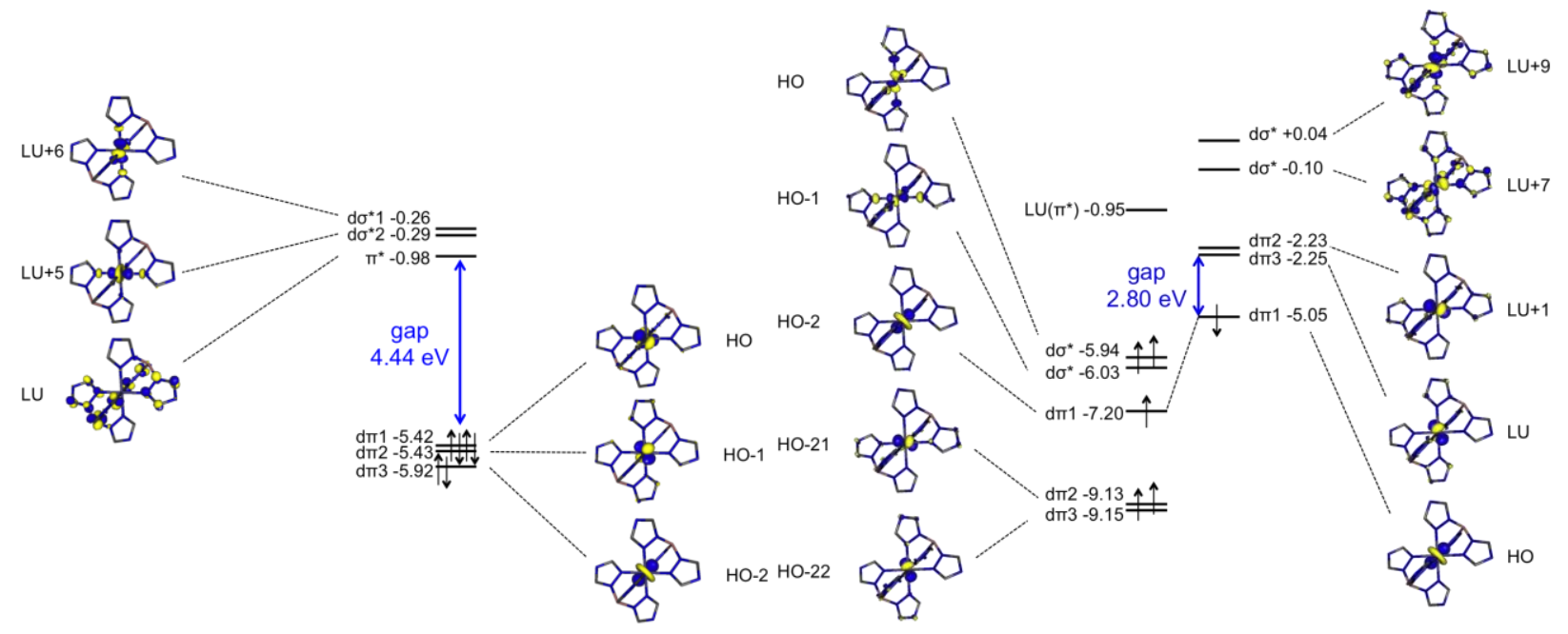

Figure 1. Kohn-Sham MO diagrams of the neutral ${ }^{1} \mathrm{LS}$ and ${ }^{5} \mathrm{HS}$ species showing the calculated HOMO-LUMO gap (single point energy calculation with $\mathrm{CPCM}-\mathrm{MeCN}$ at the vacuum optimized geometry). Selected orbitals: d block and lowest-lying ligand-based unoccupied orbital or spin-orbital (eigenvalues in $\mathrm{eV}$ ). 
Optimization of neutral low spin ( $\left.{ }^{1} \mathrm{LS}\right)$, intermediate spin $\left({ }^{3} \mathrm{IS}\right)$ and high spin $\left({ }^{5} \mathrm{HS}\right)$ states of $\mathbf{1}$ was complemented by the optimization of reduced and oxidized species of doublet, quartet and sextet spin multiplicities $\left({ }^{2} \mathrm{LS}_{\mathrm{ox}},{ }^{2} \mathrm{LS}_{\mathrm{red}},{ }^{4} \mathrm{IS}_{\mathrm{ox}},{ }^{4} \mathrm{IS}_{\text {red, }},{ }^{6} \mathrm{HS}_{\mathrm{ox}}\right.$ and $\left.{ }^{6} \mathrm{HS}_{\mathrm{red}}\right)$. This latter part of the work is crucial as it brings fundamental insights into the relaxed geometries and electronic structures of these species. (See the Supporting Information, SI, for the computed data, including geometries, energies and orbitals.)

Oxidation of the ${ }^{1} \mathrm{LS}$ species yields a doublet state after removal of one electron from a metallic non-bonding orbital, inducing slight contractions of all Fe-N bonds in the iron(III) species. On the other hand, reduction of ${ }^{1}$ LS initially occurs on the ligand-based LUMO (kinetic product) but, following relaxation, yields the ${ }^{2} \mathrm{LS}_{\text {red }}$ species with a formally reduced $\mathrm{d}^{7}$ iron(I) center (thermodynamic product) resulting from the formal addition of one electron into the LUMO +5 of ${ }^{1}$ LS. Following the optimization of two different reduction products starting from the LS state (depending on the optimization convergence criteria), the minimum energy path connecting these two species was computed using the Nudged Elastic Band method. We have previously shown that this method was indeed applicable to molecular open-shell species, provided some caution is taken in order to converge the wavefunction to the desired electronic state. ${ }^{40,41}$ In the starting state, the unpaired electron is located in a ligand-based $\pi^{*}$ orbital; in the final state, the unpaired electron is located in a metal-based $\mathrm{d} \sigma^{*}$ orbital. Consequently the relaxed geometries are clearly distinguishable, with two elongated Fe-N bonds in the final state ( $+0.45 \AA$, Table S2). The internal conversion between the two doublet states corresponds to the transfer of an electron from the ligand to the metal and is accompanied by an increase of one unit in the Mulliken spin population on Fe. This is reminiscent of the ${ }^{3} \mathrm{MLCT}-{ }^{3} \mathrm{MC}$ internal conversion process occurring for instance in $\left[\mathrm{Ru}(\mathrm{bpy})_{3}\right]^{2+} \cdot{ }^{42,43}$ In the current case, an energy 
barrier as low as $0.06 \mathrm{eV}$ was obtained (Figure 2). Therefore it would seem very difficult, if not impossible, to observe by conventional electrochemistry techniques a reduction product that would differ from the low-lying thermodynamic reduction product involving metal-centered reduction.
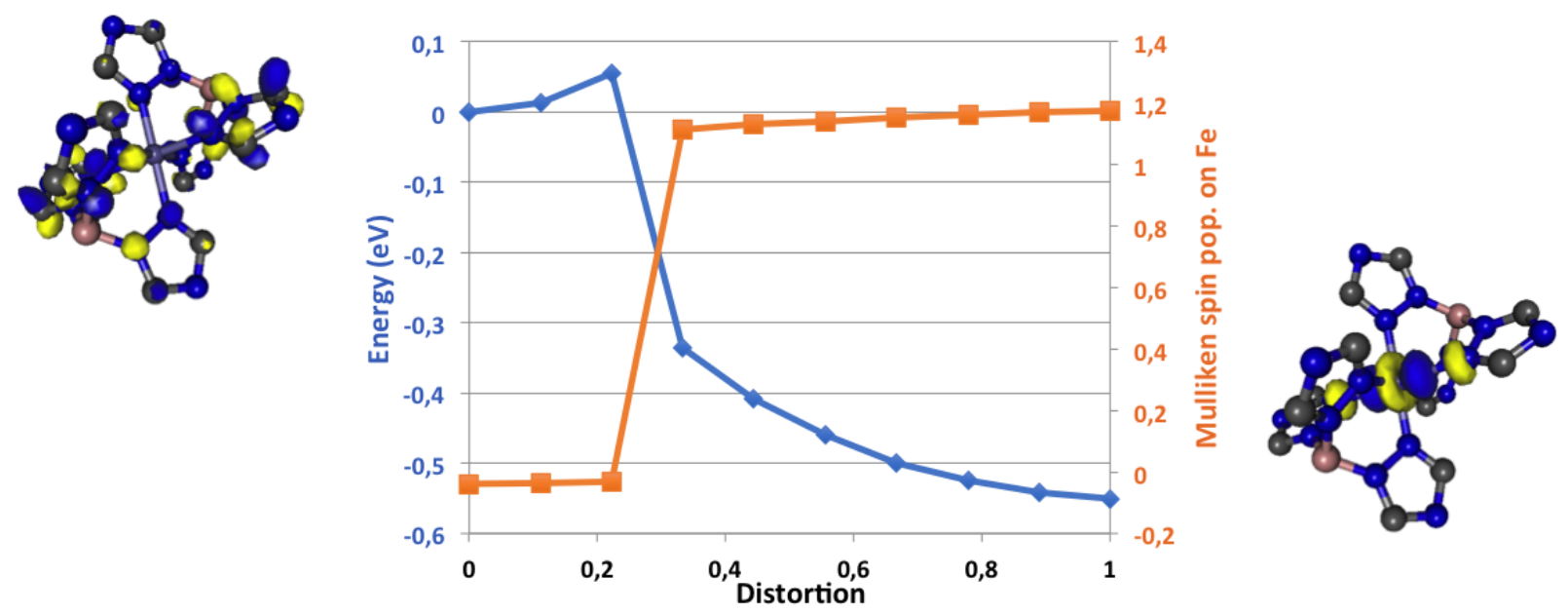

Figure 2. Minimum energy path connecting the kinetic and thermodynamic reduction products of the LS state. The $x$-axis represents the geometric distortion along the reaction coordinate: at $x$ $=0$, ligand-based reduction product; at $x=1$, metal-based reduction product (the lines are guides to the eye). Singly-occupied molecular orbital of each ${ }^{2} \mathrm{LS}_{\text {red }}$ species is shown.

Let us now examine the redox processes from ${ }^{5} \mathrm{HS}$. The least bound electron in ${ }^{5} \mathrm{HS}$ is a $\beta$ electron located in a metallic $d \pi$ orbital (eigenvalue $-5.05 \mathrm{eV}$ ). Removal of this electron leads to ${ }^{6} \mathrm{HS}_{\text {ox }}$ with contracted Fe(III)-N bonds. Alternatively, formal removal of the second least bound electron ( $\alpha$ electron located in a metallic $d \sigma^{*}$ orbital, eigenvalue $-5.94 \mathrm{eV}$ ) leads to ${ }^{4} \mathrm{IS}_{\mathrm{ox}}$. Interestingly, these two $\mathrm{Fe}(\mathrm{III})$ states are very close in energy after relaxation, ${ }^{4} \mathrm{IS}$ ox being actually $0.14 \mathrm{eV}$ lower than ${ }^{6} \mathrm{HS}_{\mathrm{ox}}$ (this value lies within the accuracy of the method). We would thus expect ${ }^{6} \mathrm{HS}_{\mathrm{ox}}$ to be the kinetic oxidation product, eventually populating ${ }^{4} \mathrm{IS}_{\mathrm{ox}}$ following spin crossover (Scheme S1). In terms of reduction, this could take place either on the metal $(\beta d \pi$ 
orbital, eigenvalue $-2.25 \mathrm{eV}$ ) to yield ${ }^{4} \mathrm{IS}_{\text {red }}$ or on the ligands ( $\alpha \pi^{*}$ orbital, eigenvalue $-0.95 \mathrm{eV}$ ) to yield ${ }^{6} \mathrm{HS}_{\text {red. }}$. In this case there is a clear energy gap between these two relaxed states (about $1 \mathrm{eV}$ ) in favor of ${ }^{4} \mathrm{IS}_{\text {red, }}$, which bears a formal $\mathrm{Fe}(\mathrm{I})$ center.

Scheme 1 gives an overview of the energetics of the different oxidation and reduction products. It must be noted that these electronic energies were obtained by performing single point energy calculations in acetonitrile solvent, at the vacuum-optimized geometries, in order to be similar to the experimental conditions of the electrochemical studies (vide infra).

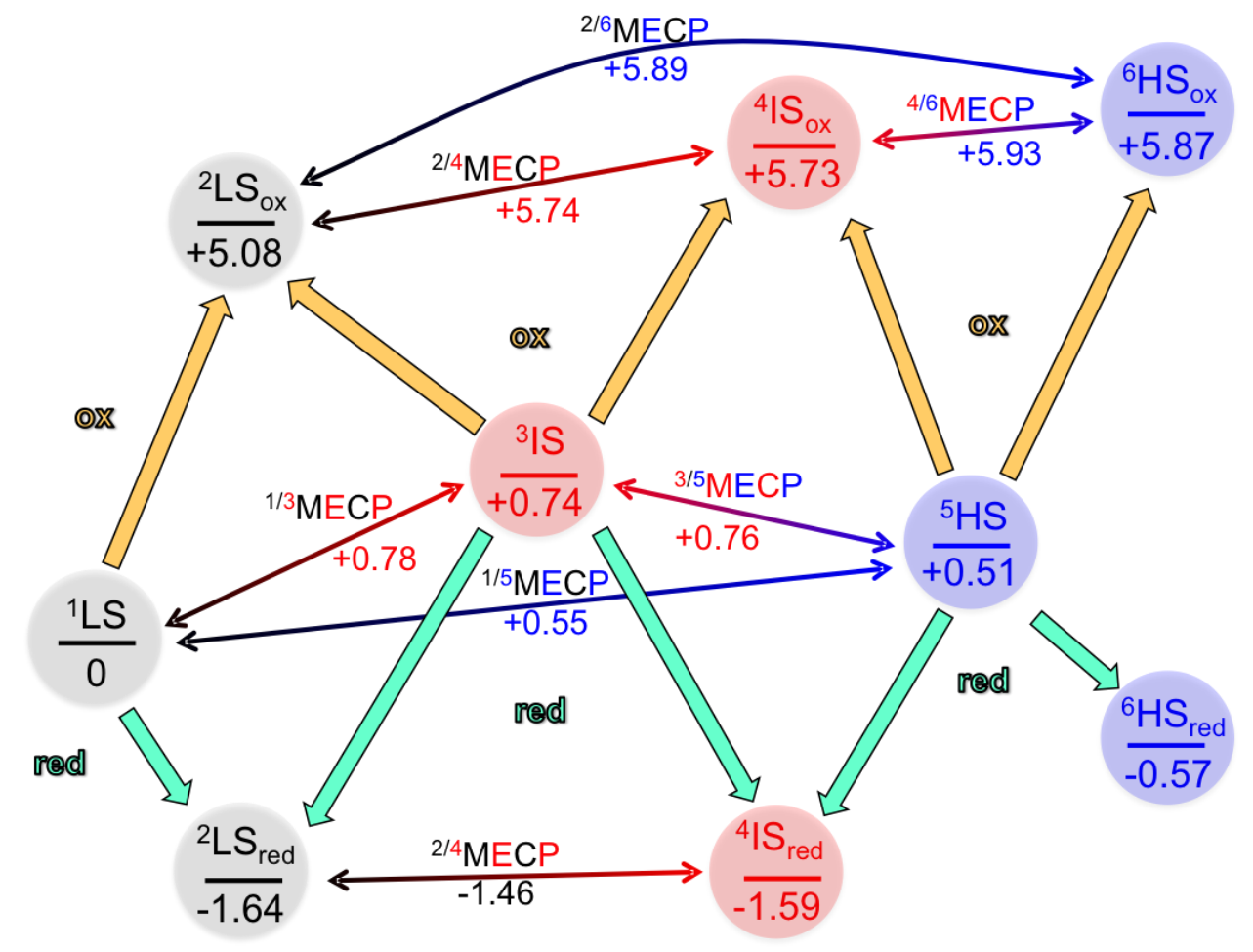

Scheme 1. Electronic energy gaps $(\mathrm{eV})$ with respect to the LS state (B3LYP* in CPCMacetonitrile). Discs stand for local minima. Arrows indicate the various connections through redox (thick arrows) or spin crossover (thin arrows) processes.

Overall, the calculations point to metal-based thermodynamic redox products in all instances. As estimated from the eigenvalue of the least bound electron (HOMO) in the parent neutral 
species $\left(-5.42 \mathrm{eV}\right.$ in ${ }^{1} \mathrm{LS} ;-5.05 \mathrm{eV}$ in $\left.{ }^{5} \mathrm{HS}\right)$, oxidation is expected to be slightly easier in the high spin species. In contrast, as estimated from the energy gaps between optimized species $\left({ }^{2} \mathrm{LS}_{\mathrm{ox}}\right.$ ${ }^{1} \mathrm{LS}: 5.08 \mathrm{eV} ;{ }^{6} \mathrm{HS}_{\mathrm{ox}}-{ }^{5} \mathrm{HS}: 5.36 \mathrm{eV}$ ), oxidation is expected to be slightly more difficult for the high-spin species. We expect the latter method, which takes into account the relaxation of the redox species, to be in better agreement with experiment. Following the same line of reasoning based on thermodynamic considerations, the reduction of ${ }^{5} \mathrm{HS}$ is expected to be easier than the reduction of ${ }^{1}$ LS. As indicated in Scheme 1 (see also Scheme S1 in the SI), minimum energy crossing points (MECP) have been optimized in the neutral, cationic and anionic species as their energies and geometries inform us on the ease for the system to undergo spin crossover. It turns out that all MECPs are very close in energy and geometry to one of the neighboring minima $(<0.1 \mathrm{eV})$. As a result, spin crossover processes are expected to be easy whatever the starting spin state and whatever the charge.

In order to assess the validity of our calculations we have conducted a cyclic voltammetry investigation of the redox properties of compound $\mathbf{1}$ at two different temperatures. Details of the $\mathrm{CV}$ experiments and results are given in the SI. Briefly, the electrochemical oxidation of 1 in acetonitrile involves a reversible one-electron transfer that may confidently be ascribed as an $\mathrm{Fe}(\mathrm{III}) / \mathrm{Fe}(\mathrm{II})$ redox process with a half-wave potential of $\mathrm{E}_{1 / 2}=+1.01 \mathrm{~V}$ vs. SHE (standard hydrogen electrode) at $298 \mathrm{~K}$ and $+1.09 \mathrm{~V}$ vs. SHE at $343 \mathrm{~K}$. On the other hand, the reduction of 1 turned out to be electrochemically irreversible and very sensitive to the experimental details. For this reason, we restrict the following discussion to the oxidation process. In acetonitrile, $\mathbf{1}$ displays a gradual spin conversion with an equilibrium temperature of $\sim 293 \mathrm{~K}$ (as estimated from temperature-dependent UV-vis absorption spectroscopy, Figure S5). In our experimental conditions, the proportion of LS species drops from $50 \%$ at room temperature to $10 \%$ at high 
temperature. This means that the HS and LS species are in equilibrium with fast interconversion rates in the whole temperature range of the $\mathrm{CV}$ experiments, which prevents the assessment of the redox potential of both species. We can assume that the observed process corresponds to the oxidation of the most oxidizable species, i.e. the LS species according to the DFT results. As discussed in ref. 15, in coupled oxidation and spin-exchange processes, the oxidation potential can be written as:

$$
\mathrm{E}_{\mathrm{ox}}^{\circ} \approx \mathrm{E}_{\mathrm{LS}}^{\circ}-(\mathrm{RT} / \mathrm{F}) \ln \left(\mathrm{n}_{\mathrm{LS}}\right)
$$

where $n_{H S}$ and $n_{L S}$ are the temperature dependent HS and LS fractions, respectively, $R$ is the molar gas constant and $F$ is the Faraday constant $\left(\mathrm{n}_{\mathrm{LS}}{ }^{298 \mathrm{~K}}=0.5, \mathrm{n}_{\mathrm{LS}}{ }^{343 \mathrm{~K}}=0.1\right)$. Thus eq. (1) predicts an increase of ca. $+50 \mathrm{mV}$ of the oxidation potential when going from 25 to $70^{\circ} \mathrm{C}$, which is indeed very close to the experimentally observed value of $+80 \mathrm{mV}$. This discrepancy could be related to solubility issues, which contribute to the temperature dependence of $E_{1 / 2}$. Then we can estimate the value of $\mathrm{E}_{\mathrm{LS}}^{\circ}=1.03 \mathrm{~V}$ vs. SHE. Taking into account the literature value of Gibbs free energy change of SHE $\left(\Delta \mathrm{G}^{\circ}{ }_{\mathrm{SHE}}=-4.28 \mathrm{eV}\right)^{44}$ and the Gibbs free energy change for the oxidation of the LS species (reoptimized in solvent, $\Delta \mathrm{G}_{\mathrm{LS} / \mathrm{LSox}}^{1}=5.09 \mathrm{eV}$, Table $\mathrm{S} 4$ ) the calculations give $\mathrm{E}_{\mathrm{LS}}^{\circ}=0.8 \mathrm{~V}$ vs. $\mathrm{SHE}$, in reasonably good agreement with the experiment. Additionally, reoptimization of ${ }^{5} \mathrm{HS},{ }^{6} \mathrm{HS}_{\mathrm{ox}}$ and ${ }^{4} \mathrm{IS}_{\mathrm{ox}}$ in solvent confirms the degeneracy of the quartet and sextet oxidized species, and confirms that the high spin complex should be more difficult to oxidize $\left(\Delta \mathrm{G}_{\mathrm{HS} / \mathrm{HSox}}^{5}=5.46 \mathrm{eV}, \Delta \mathrm{G}_{\mathrm{HS} / \mathrm{ISox}}^{5}=5.43 \mathrm{eV}\right.$, Table S4) than the low spin complex.

\section{Conclusion}


In summary, through a detailed DFT study of the oxidation and reduction processes in the spin crossover complex $\left[\mathrm{Fe}\left(\mathrm{HB}(1,2,4-\text { triazol-1-yl })_{3}\right)_{2}\right]$, we have shown that there is no unequivocal connection between the frontier molecular orbital diagram and MO eigenvalues of the neutral molecule and the redox potentials in the two spin states. The effects of electronic repolarization and geometric relaxation in the oxidized/reduced species, as well as spin-state equilibria in the different oxidation states, turned out to be substantial on the localization of the redox events, hence on the electronic structures and final relaxed geometries, explaining why considering solely the HOMO and LUMO of the parent species can be misleading, particularly in spin crossover complexes. As an illustration, during the course of the calculations, we have encountered what can be seen as kinetic oxidation or reduction products as well as a thermodynamic oxidation or reduction products. As such, the computational results obtained here allow to shed light on some aspects that have not received enough attention in the rationalization of combined charge transfer and spin crossover phenomena, which are relevant not only for SCO-based electronic junctions, but also for a variety of biochemical, catalytic and energy storage/conversion processes.

\title{
ASSOCIATED CONTENT
}

Supporting Information. The following files are available free of charge. Experimental and computational details. Supplementary CV, UV-VIS and DFT data (PDF).

\author{
AUTHOR INFORMATION
}

Corresponding Authors 
* isabelle.dixon@irsamc.ups-tlse.fr, azzedine.bousseksou@lcc-toulouse.fr

\section{Notes}

The authors declare no competing financial interest.

\section{ACKNOWLEDGMENT}

HPC resources at LCPQ are gratefully acknowledged, as well as discussions with F. Alary and

J.-L. Heully.

\section{REFERENCES}

1. Gütlich, P.; Hauser, A.; Spiering, H. Thermal and Optical Switching of Iron(II) Complexes. Angew. Chem., Int. Ed. Engl. 1994, 33, 2024-2054.

2. Bousseksou, A.; Molnar, G.; Salmon, L.; Nicolazzi, W. Molecular Spin Crossover Phenomenon: Recent Achievements and Prospects. Chem. Soc. Rev. 2011, 40, 3313-3335.

3. Lefter, C.; Davesne, V.; Salmon, L.; Molnár, G.; Demont, P.; et al. Charge Transport and Electrical Properties of Spin Crossover Materials: Towards Nanoelectronic and Spintronic Devices. Magnetochemistry 2016, 2, 18.

4. Kumar, K. S.; Ruben, M. Emerging trends in spin crossover (SCO) based functional materials and devices. Coord. Chem. Rev. 2017, 346, 176.

5. Molnár, G.; Rat, S.; Salmon, L.; Nicolazzi, W.; Bousseksou, A. Spin Crossover Nanomaterials: From Fundamental Concepts to Devices. Adv. Mater. 2018, 30, 1703862.

6. Bellec, A.; Lagoute, J.; Repain, V. Molecular electronics: Scanning tunneling microscopy and single-molecule devices, C. R. Chim. 2018, 21, 1287-1299.

7. Ruiz, E. Charge transport properties of spin crossover systems. Phys. Chem. Chem. Phys. 2014, 16, 14-22.

8. Rosseinsky, D. R.; Tonge, J. S. Temperature Dependence of Dielectric Relaxation and Conductivity in Mixed-valence Potassium Manganate-Permanganate. J. Chem. Soc., Faraday Trans. 1982, 78, 3595-3603.

9. McCreery, R. L.; Yan, H.; Bergren, A. J. A critical perspective on molecular electronic junctions: there is plenty of room in the middle. Phys. Chem. Chem. Phys. 2013, 15, 10651081.

10. Baadji, N.; Sanvito, S. Giant resistance change across the phase transition in spin-crossover molecules. Phys. Rev. Lett. 2012, 108, 217201. 
11. Aravena, D.; Ruiz, E. Coherent transport through spin-crossover single molecules. J. Am. Chem. Soc. 2012, 134, 777-779.

12. Jasper-Toennies, T.; Gruber, M.; Karan, S.; Jacob, H.; Tuczek, F.; Berndt, R. Robust and Selective Switching of an $\mathrm{Fe}^{\mathrm{III}}$ Spin-Crossover Compound on $\mathrm{Cu}_{2} \mathrm{~N} / \mathrm{Cu}(100)$ with Memristance Behavior, Nano Lett. 2017, 17, 6613-6619.

13. Miyamachi, T.; Gruber, M.; Davesne, V.; Bowen, M.; Boukari, S.; et al. Robust Spin Crossover and Memristance Across a Single Molecule. Nat. Commun. 2012, 3, 938.

14. Vela, S.; Verot, M.; Fromager, E.; Robert, V. Electron transport through a spin crossover junction. Perspectives from a wavefunction-based approach. J. Chem. Phys. 2017, 146, 064112 .

15. Turner, J. W.; Schultz, F. A. Coupled electron-transfer and spin-exchange reactions. Coord. Chem. Rev. 2001, 219-221, 81-97.

16. The Biological Chemistry of Iron, Dunford, H. B.; Dolphin, D.; Raymond, K. N.; Sieker, L. Eds); NATO Advanced Study Institute Series, Vol. 89, Reidel Publishing Company, Dordrecht, Holland, 1982.

17. Sligar, S. G.; Egeberg, K. D.; Sage, J. T.; Morikis, D.; Champion, P. M. Alteration of Heme Axial Ligands by Site-Directed Mutagenesis: A Cytochrome Becomes a Catalytic Demethylase, J. Am. Chem. Soc. 1987, 109, 7896-7897.

18. Scheidt, W. R.; Reed, C. A. Spin-state/stereochemical relationships in iron porphyrins: implications for the hemoproteins. Chem. Rev. 1981, 81, 543-555.

19. Watanabe, E.; Zhao, W.; Sugahara, A.; Mortemard de Boisse, B.; Lander, L. et al. RedoxDriven Spin Transition in a Layered Battery Cathode Material. Chem. Mater. 2019, 31, $2358-2365$.

20. Yang, C.; Nikiforidis, G.; Park, J. Y.; Choi, J.; Luo, Y.; et al. Designing Redox-Stable Cobalt-Polypyridyl Complexes for Redox Flow Batteries: Spin-Crossover Delocalizes Excess Charge. Adv. Energy Mater. 2018, 8, 1702897.

21. Emery, N.; Sougrati, M. T.; Panabière, E.; Bacha, S.; Fraisse, B.; et al. Unidimensional unit cell variation and $\mathrm{Fe}^{+3} / \mathrm{Fe}^{+4}$ redox activity of $\mathrm{Li}_{3} \mathrm{FeN}_{2}$ in $\mathrm{Li}$-ion batteries. J. Alloys Compd. 2017, 696, 971-979.

22. Mosconi, E.; Yum, J.-H.; Kessler, F.; Gomez Garcia, C. J.; Zuccaccia, C.; et al. Cobalt Electrolyte/Dye Interactions in Dye-Sensitized Solar Cells: A Combined Computational and Experimental Study, J. Am. Chem. Soc. 2012, 134, 19438-19453.

23. Ashley, D. C.; Jakubikova, E. Tuning the Redox Potentials and Ligand Field Strength of $\mathrm{Fe}(\mathrm{II})$ Polypyridines: The Dual $\pi$-Donor and $\pi$-Acceptor Character of Bipyridine. Inorg. Chem. 2018, 57, 9907-9917.

24. Sligar, S. G.; Cinti, D. L. Gibson, G. G.; Schenkman, J. B. Spin state control of the hepatic cytochrome P450 redox potential. Biochem. Biophys. Res. Commun. 1979, 90, 925-932. 
25. Shankar, S.; Peters, M.; Steinborn, K.; Krahwinkel, B.; Sonnichsen, F. D. et al. Lightcontrolled switching of the spin state of iron(III). Nat. Commun. 2018, 9, 4750.

26. Kadish, K. M.; Das, K.; Schaeper, D.; Merrill, C. L.; Welch, B. R.; Wilson, L. J. Spin State Dependent Redox Properties of the $\left[\mathrm{Fe}^{\mathrm{III}}(\mathrm{X}-\mathrm{Sal})_{2} \text { trien }\right]^{+}$Spin-Equilibrium System in Solution. Inorg. Chem. 1980, 19, 2816-2821.

27. Kadish, K. M.; Su, C. H.; Wilson, L. J. Spin-State Dependence of Heterogeneous ElectronTransfer Rates for the $\left[\mathrm{Fe}^{\mathrm{III}}(\mathrm{X}-\mathrm{Sal})_{2} \text { trien }\right]^{+}$Spin-Equilibrium System in Solution. Inorg. Chem. 1982, 21, 2312-2314.

28. Kadish, K. M.; Su, C. H. Relationships between Electron-Transfer Rate Constants of Bis(ligated)(octaethylporphinato)iron(III) Perchlorate and the Presence of a Spin Equilibria. J. Am. Chem. Soc. 1983, 105, 177-180.

29. Zhu, T.; Su, C.-H.; Schaeper, D.; Lemke, B. K. ; Wilson, L. J. ; Kadish, K. M. Solvent and Structure Effects on the Spin-Crossover and Electron-Transfer Properties of $\left[\mathrm{Fe}^{\mathrm{III}}(\mathrm{X}-\right.$ Salmeen $\left.)_{2}\right]\left(\mathrm{PF}_{6}\right)$ and $\left[\mathrm{Fe}^{\mathrm{III}}(\mathrm{X}-\mathrm{Sal})_{2}\right.$ trien $]\left(\mathrm{PF}_{6}\right)$ Complexes. Inorg. Chem. 1984, 23, 43454349.

30. Brady, C.; McGarvey, J. J.; McCusker, J. K.; Toftlund, H.; Hendrickson, D. N. TimeResolved Relaxation Studies of Spin Crossover Systems in Solution. Top. Curr. Chem. 2004, 235, 1-22.

31. Paulsen, H.; Schunemann, V.; Wolny, J. Progress in Electronic Structure Calculations on Spin-Crossover Complexes. Eur. J. Inorg. Chem. 2013, 628-641.

32. Lawson Daku, L. M. Modeling Transition Metal Complexes in the Framework of the SpinCrossover Phenomenon: A DFT Perspective. Curr. Inorg. Chem. 2013, 3, 242-259.

33. Cirera, J.; Via-Nadal, M.; Ruiz, E. Benchmarking Density Functional Methods for Calculation of State Energies of First Row Spin-Crossover Molecules. Inorg. Chem. 2018, 57, 14097-14105.

34. Kepp, K. P. Theoretical Study of Spin Crossover in 30 Iron Complexes. Inorg. Chem. 2016, 55, 2717-2727.

35. Reiher, M. Theoretical Study of the Fe(phen $)_{2}(\mathrm{NCS})_{2}$ Spin-Crossover Complex with Reparametrized Density Functionals, Inorg. Chem. 2002, 41, 6928-6935.

36. Ashley, D. C.; Jakubikova, E. Ironing out the photochemical and spin-crossover behavior of $\mathrm{Fe}(\mathrm{II})$ coordination compounds with computational chemistry. Coord. Chem. Rev. 2017, $337,97-111$.

37. Janiak, C.; Scharmann, T. G.; Green, J. C.; Parkin, R. P. G.; Kolm, M. J.; et al. Effects of Nitrogen Substitution in Poly(pyrazoly1)borato Ligands : From Orbital Energy Levels to C-H ${ }^{\cdots}$ O Hydrogen Bonding. Chem. Eur. J. 1996, 2, 992-1000. 
38. Shalabaeva, V.; Ridier, K.; Rat, S.; Manrique-Juarez, M. D. ; Salmon, L. ; et al. Room temperature current modulation in large area electronic junctions of spin crossover thin films. Appl. Phys. Lett. 2018, 112, 013301.

39. Poggini, L.; Gonidec, M.; Gonzalez-Estefan, J. H.; Pecastaings, G.; Gobaut, B.; et al. Vertical Tunnel Junction Embedding a Spin Crossover Molecular Film, Adv. El. Mater. 2018, 4, 1800204.

40. Göttle, A. J.; Alary, F.; Boggio-Pasqua, M.; Dixon, I. M.; Heully, J.-L.; et al. Pivotal Role of a Pentacoordinate ${ }^{3} \mathrm{MC}$ State on the Photocleavage Efficiency of a Thioether Ligand in Ruthenium(II) Complexes: A Theoretical Mechanistic Study. Inorg. Chem. 2016, 55, 4448-4456.

41. Dixon, I. M.; Heully, J.-L.; Alary, F.; Elliott, P. I. P. Theoretical illumination of highly original photoreactive ${ }^{3} \mathrm{MC}$ states and the mechanism of the photochemistry of Ru(II) tris(bidentate) complexes. Phys. Chem. Chem. Phys. 2017, 19, 27765-27778.

42. Soupart, A.; Dixon, I. M.; Alary, F.; Heully, J.-L. DFT rationalization of the room- temperature luminescence properties of $\mathrm{Ru}(\mathrm{bpy})_{3}{ }^{2+}$ and $\mathrm{Ru}(\mathrm{tpy})_{2}{ }^{2+}:{ }^{3} \mathrm{MLCT}-{ }^{3} \mathrm{MC}$ minimum energy path from NEB calculations and emission spectra from VRES calculations. Theor. Chem. Acc. 2018, 137, 37.

43. Soupart, A. ; Alary, F. ; Heully, J.-L. ; Elliott, P. I. P. ; Dixon, I. M. Exploration of Uncharted ${ }^{3} \mathrm{PES}$ Territory for $\left[\mathrm{Ru}(\mathrm{bpy})_{3}\right]^{2+}$ : A New ${ }^{3} \mathrm{MC}$ Minimum Prone to Ligand Loss Photochemistry. Inorg. Chem. 2018, 57, 3192-3196.

44. Kelly, C. P.; Cramer, C. J.; Truhlar, D. G. Aqueous solvation free energies of ions and ionwater clusters based on an accurate value for the absolute aqueous solvation free energy of the proton. J. Phys. Chem. B 2006, 110, 16066-16081. 


\section{Table of Contents}

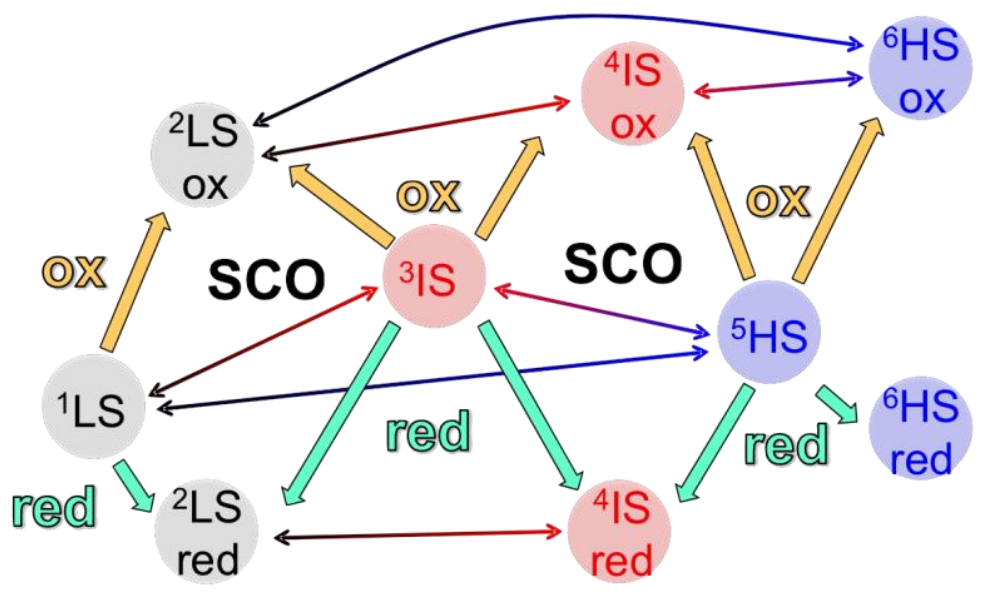

\section{Synopsis}

In a joint DFT and experimental study, we have explored the interplay between redox and spin crossover processes in $\left[\mathrm{Fe}\left(\mathrm{HB}(1,2,4 \text {-triazol-1-yl })_{3}\right)_{2}\right]$ in $\mathrm{MeCN}$ solution $\left(25\right.$ and $\left.70^{\circ} \mathrm{C}\right)$. The electronic structure of all spin and redox species is reported. This is required to decipher the variety of processes occurring upon removal or addition of an electron to the complex. This work highlights that a simple picture based on the HOMO-LUMO levels of the neutral HS and LS species cannot provide even a qualitative link between redox potential and spin state of the complex. 